\title{
EFFICIENT NUMERICAL CONTINUATION AND STABILITY ANALYSIS OF SPATIOTEMPORAL QUADRATIC OPTICAL SOLITONS *
}

\author{
D.J.B. LLOYD ${ }^{\dagger}$ AND A.R. CHAMPNEYS $\ddagger$
}

\begin{abstract}
A numerical method is set out which efficiently computes stationary (z-independent) two- and three-dimensional spatiotemporal solitons in second-harmonic-generating media. The method relies on a Chebyshev decomposition with an infinite mapping, bunching the collocation points near the soliton core. Known results for the type-I interaction are extended and a stability boundary is found by two-parameter continuation as defined by the Vakhitov-Kolokolov criteria. The validity of this criteria is demonstrated in (2+1)-dimensions by simulation and direct calculation of the linear spectrum. The method has wider applicability for general soliton-bearing equations in (2+1)- and (3+1)-dimensions.
\end{abstract}

Key words. Continuation, Optical Soliton, Second-harmonic-generation, type-I interaction.

AMS subject classifications. $37 \mathrm{~K} 50,65 \mathrm{~N} 35,65 \mathrm{P} 40,74 \mathrm{~S} 25$

1. Introduction. Optical spatiotemporal solitons in second-harmonic-generating media have received much attention due to the possibility of creating fully localised, self-supporting objects which freely propagate in a nonlinear medium [12]. Unlike Kerr media, it has been shown that there is no wave collapse in $(2+1)$ and $(3+1) \mathrm{D}$ in quadratic $\left(\chi^{2}\right)$ media. The equations governing the evolution of these quadratic solitons are non-integrable so that analytical closed form stable solutions do not exist. For a review of solitons due to quadratic nonlinearities see [4].

Malomed et al. [16] studied general stationary, non-radially symmetric solitons found in the type-I interaction second harmonic generation (SHG) case. Their approach was to first construct a Gaussian variational approximation of solitons in $(2+1) \mathrm{D}$ and $(3+1) \mathrm{D}$ and analytically find an approximate stability boundary. They then carried out numerical simulations of the full z-dependent equations to ascertain the accuracy of the approximate stability boundary. Since this Gaussian approximation amounts to a one-mode Galerkin truncation, the stability boundary was found to be 'rather fuzzy'. Part of the problem is that the accuracy of this approximation deteriorates with increasing dimensionality due to the approximation 'cutting off' the tails of the soliton.

A more thorough analysis of stationary solitons in type-I interaction was carried out by Mihalache et al. [17] who considered the numerical boundary value problem (BVP) for the stationary light bullets. Existence and stability of 'elliptic' solitons was explored in $(2+1) \mathrm{D}$ and $(3+1) \mathrm{D}$ with and without 'walkoff' $[17,18]$. Later, Towers et al. [24] explored the region where the second harmonic dispersion is normal via solving the initial value problem starting from an initial Gaussian pulse. They also showed analytically that solitons with small oscillating tails at infinity, known as 'quasi-solitons', should exist in this region, though they were unable to find any numerically. One of the key problems with both numerical approaches is the high computational effort required to solve the equations with either finite-differences or Fourier spectral methods. In both cases supercomputers were required to reduce the computational time.

The object of this work is to provide an efficient and simple numerical method for calculating stationary spatiotemporal solitons, not necessarily just in the second-harmonic-generation equations, but also in other soliton bearing equations in $(2+1) \mathrm{D}$ and $(3+1) \mathrm{D}$. There are several benefits with using our method. Firstly, we can easily move through parameter space and between $(2+1) \mathrm{D}$ and $(3+1) \mathrm{D}$, assuming radial symmetry in $(3+1) D$. Secondly, we can accurately compute light bullets with a mesh in the order of tens of modes rather than hundreds, greatly reducing the computational effort. Thirdly, this approach also allows us to embed the method in a numerical continuation environment, e.g. AUTO [9], to compute branches of the solutions. Fourthly, and perhaps most crucially, using criteria such as that due to Vakhitov and Kolokolov which define soliton instabilities [21], one can then append the stability criteria to the numerical continuation problem. The Vakhitov-Kolokolov (VK) condition states that solitons will become unstable if the gradient of the energy of the solution $(Q)$ as the effective propagation constant $(\kappa)$ varies, becomes negative (i.e

\footnotetext{
${ }^{*}$ This work was supported by an EPSRC Research Studentship.

$\dagger$ Bristol Centre for Applied Nonlinear Mathematics,Department of Engineering Mathematics, University of Bristol, Queen's Building, University Walk, Bristol BS8 1TR, UK (david.1loyd@bris.ac.uk).

$\ddagger$ Bristol Centre for Applied Nonlinear Mathematics, Department of Engineering Mathematics, University of Bristol, Queen’s Building, University Walk, Bristol BS8 1TR, UK (a.r.champneys@bris .ac.uk)
} 
$\partial_{\kappa} Q<0$ ). A stability boundary of the solutions corresponds to when the gradient is zero (i.e. $\partial_{\kappa} Q=0$ ). In a continuation environment this corresponds to a fold in the energy of the solution as the effective propagation constant is varied. Thus we can trace out stability boundaries automatically and accurately with the kind of computational effort that takes minutes on a modern PC.

Finally, criteria such as the VK are only necessary conditions for stability. The sufficiency of this condition can also be checked by computation of the spectrum linearised about the localised solution, see Mihalache et al. [17] for confirmation of the validity of the VK criteria in $(2+1)$ - and (3+1)-dimensions. We demonstrate that an adaption of our Chebyshev method can be used to calculate the linear spectrum. The description in terms of only tens of modes helps make such computations feasible.

The paper is organised in the following way. In section 2 we set out the problem. The numerical scheme for the computation of stationary solitons and their stability is stated in section 3. Our results for the type-I SHG interaction equations are presented in section 4. Section 5 draws conclusions and suggests avenues for future work.

2. Problem statement. We are interested in stationary (z-independent) soliton solutions to the scaled equations describing co-propagation of the fundamental harmonic (FH) and second harmonic (SH) field in a quadratic optical medium,

$$
\begin{aligned}
i u_{z}+\nabla_{\rho}^{2} u+u_{\tau \tau}-u+v \bar{u} & =0 \\
2 i v_{z}+\nabla_{\rho}^{2} v+\delta v_{\tau \tau}-\gamma v+\frac{1}{2} u^{2} & =0
\end{aligned}
$$

(see [11] for their derivation and physical meaning).

Here $u=\sqrt{2} \Phi_{1} e^{-i z / z_{0}} z_{0} \omega_{1}^{2} \chi^{(2)} /\left(k_{1} c^{2}\right)$ and $v=\Phi_{2} e^{-2 i z / z_{0}} z_{0} \omega_{1}^{2} \chi^{(2)} /\left(k_{1} c^{2}\right), \Phi_{1,2}$ are complex electricfield envelopes at the frequencies $\omega_{1}$ and $2 \omega_{1}, k_{1} \equiv k\left(\omega_{1}\right)$ is the FH carrier wave number. The normalised propagation distance and reduced time are $\xi=z / z_{0}$ and $\tau=\left(t-z / \nu_{g}\right) / t_{0}$, respectively, with $\nu_{g}$ and $t_{0}$ being the group velocity and time scale at $\mathrm{FH} . z_{0}=2 t_{0}^{2} / k_{1}^{\prime \prime}$, where $k_{1}^{\prime \prime}$ is the group-velocity-dispersion (GVD) coefficient at FH, and the non-dimensional transverse radial co-ordinate is $\rho=\sqrt{x^{2}+y^{2}} / r_{0}$ where $r_{0}^{2}=\left(z_{0} / 2 k_{1}\right)$. The diffraction operator, $\nabla_{\rho}^{2} \equiv \partial^{2} / \partial \rho^{2}+[(D-1) / \rho] \partial / \partial \rho$, where $D+1=2$ or 3 is the spatial dimension, acts on the transverse spatial co-ordinate(s) (we shall assume cylindrical symmetry in $(3+1)$-dimensions). The phase mismatch between the harmonics $\gamma=4+2 z_{0}\left[2 k\left(\omega_{1}\right)-k\left(2 \omega_{1}\right)\right]$ and the ratio of the SH and FH GVD coefficients is $\delta=k_{2}^{\prime \prime} / k_{1}^{\prime \prime}$.

Under the substitution, $u(z, \rho, \tau)=u(\rho, \tau) e^{i \kappa z}, v(z, \rho, \tau)=v(\rho, \tau) e^{2 i \kappa z}$, where $\kappa$ is the effective propagation constant, we seek stationary, $z$-independent soliton solutions defined on the infinite domain, $-\infty<$ $\rho<\infty$ and $-\infty<\tau<\infty$, given by the following equations for the real functions $u$ and $v$ :

$$
\begin{aligned}
u_{\rho \rho}+(d-1) \rho^{-1} u_{\rho}+u_{\tau \tau}-(1+\kappa) u+v u & =0, \\
v_{\rho \rho}+(d-1) \rho^{-1} v_{\rho}+\delta v_{\tau \tau}-(\gamma+4 \kappa) v+\frac{1}{2} u^{2} & =0 .
\end{aligned}
$$

Equations (2.2) are known to support localised solutions, known as solitary waves, that are able to survive strong perturbations and remain intact after collisions with other solitary waves [3]. Exact solitary wave solutions to equations (2.1) are not available, except in special degenerate limits, since the equations are non-integrable and so numerical methods provide the only way to accurately calculate the solutions. Since the solitary waves seen in (2.1) have similar soliton shapes to solutions of integrable systems, we shall follow the physics convention and use the word 'soliton' interchangeably with solitary wave.

We are seeking physical localised solutions of (2.1) which correspond to the solutions that decay (exponentially) to zero at infinity. We can see from the linearisation of the equations (2.2) at infinity, that exponentially decaying solutions in both harmonics forming the soliton exist if the effective propagation constant, $\kappa>\max (-1,-\gamma / 4)$ and the GVD ratio coefficient $\delta \geq 0$. This last constraint corresponds to anomalous or zero dispersion in the second-harmonic. So we compute on a 'large' domain and apply zero Dirichlet boundary conditions at the far-field edge of the domain. If the group-velocity-dispersion is normal at the second-harmonic $(\delta<0)$, Towers et al. [24] showed analytically there should be a non vanishing, oscillating tails in the second harmonic $v$ as $\tau \rightarrow \infty$. 
3. Numerical approach. Our numerical method is based on setting up the boundary value problem of equations (2.2) and using numerical continuation software e.g. AUTO [9], to explore the parameter space of solitons. Two problems need to be tackled for this approach to work. The first is to efficiently discretise the problem in either the $\rho$ or $\tau$ co-ordinate. A straight Fourier or finite-differences discretisation scheme, though possible, has been found to require a large number of modes and hence a huge amount of computing power is required to solve the resulting ODE system (both Towers et al. [24] and Mihalache et al. [17] cite the use of supercomputers). The reason for the inefficiency is due to interpolation points being equi-spaced, so a large number of these points are required to interpolate the solution near the edge of the computational domain where the soliton is zero. A more efficient approach is to reduce the number of interpolation points near the edge of the domain and move them to the centre of the stationary soliton, where they can increase the accuracy of the method. To do this we use an adapted mesh to the localised soliton problem. Effectively, we map the spatial co-ordinate to a new scaled space and equidistribute. A similar approach to homoclinic orbits in 1D was proposed by Liu et al. [13]. Another property of these solitons is that they are smooth and infinitely differentiable. For these type of problems, spectral methods provide exponential accuracy (unlike finite-differences which is only algebraic accuracy) and so only 10s of modes should be required to accurately interpolate the soliton. The second problem is to find good solution data at a certain fixed $D, \delta, \gamma$ parameters, for numerical continuation code to get started.

We shall tackle both of these problems in the next two sub-sections. In the third section we describe how we can adapt our numerical method to compute the stability of the solitons using the Vakhitov-Kolokolov (VK) criteria and computation of the linear spectrum.

3.1. Numerical Continuation. Using an adapted mesh to solve soliton bearing equations is not a new idea. Boyd [1] talks about rational Chebyshev functions and other possible spectral functions for soliton equations including: Hermite, Whittaker cardinal and Fourier domain truncation functions. The biggest advantage with using Fourier or Chebyshev basis is that their functions and their corresponding collocation points have simple representations as well as there existing a fast transform between the physical and spectral domains. Boyd [2] shows how the Chebyshev polynomials are just Fourier cosine functions in disguise and how a mapping can be used to adapt the mesh to the localised problem while still maintaining spectral accuracy.

There are three approaches one can take to implement the rational Chebyshev method: the discreteFourier-transform (DFT), the fast Fourier transform (FFT), and collocation differentiation matrices [25]. We have implemented all three methods and have found that they all produce the same results at roughly the same speed for truncations in the order of $10 \mathrm{~s}$ of modes. However, we have found that the collocation differentiation matrices approach to be the most simple to program, and hence to be the method of choice in practice. Henceforth, we describe only that method applied to equations (2.2). Note the approach is quite general and is easily extended to other systems.

For the problem at hand, we choose to discretise in the $\tau$ co-ordinate since we wish to explore the $\delta=0$ region and discretising in $\rho$ would make the problem singular at $\delta=0$. To discretise in $\tau$ we expand $u$ and $v$, such that:

$$
\begin{aligned}
& u_{i}=u\left(\rho, \tau_{i}=f\left(x_{i}\right)\right), \\
& v_{i}=v\left(\rho, \tau_{i}=f\left(x_{i}\right)\right), \quad i=0 \ldots N,
\end{aligned}
$$

where $x \in[-1,1]$ is the computational Chebyshev co-ordinate and $f$ is a function that maps the infinite domain $[-\infty, \infty]$ to $[-1,1]$. There two standard choices for such mapping $([2,5])$ : the algebraic map

$$
f(x)=L \frac{x}{\sqrt{1-x^{2}}},
$$

and the exponential map

$$
f(x)=L \tanh ^{-1} x .
$$

The constant $L$ sets the length scale of the map and it can vary anywhere between 0 and $+\infty$; with the collocation points being more 'bunched' near the origin for small $L$. In general, if the solitons decay exponentially then the exponential map will be the most efficient but it is also the most sensitive to changes 
in the mapping constant $L$; see Boyd [2]. Some experimentation of various length scales and number of collocation points is required to find a reasonable mapping. We shall stick with the exponential map in this paper since we shall mostly be exploring a parameter region where soliton tails decay exponentially.

Differentiation in $\tau$ is carried out using the chain rule for Chebyshev differentiation matrices [25] so that

$$
\begin{aligned}
\left(u_{\tau \tau}\right)_{i} & =\left[\left(\frac{\partial^{2} f^{-1}\left(\tau_{i}\right)}{\partial \tau^{2}} D_{N}^{(1)}+\left[\frac{\partial f^{-1}\left(\tau_{i}\right)}{\partial \tau}\right]^{2} D_{N}^{(2)}\right) u\left(\rho, x_{i}\right), \quad i=0 \ldots N\right] \\
& \equiv A_{N} u\left(\rho, x_{i}\right), \quad i=0 \ldots N,
\end{aligned}
$$

where $f^{-1}(\tau)$ is the inverse of the infinite mapping (3.3), $A_{N}=\partial^{2} f^{-1}\left(\tau_{i}\right) / \partial \tau^{2} D_{N}^{(1)}+\left[\partial f^{-1}\left(\tau_{i}\right) / \partial \tau\right]^{2} D_{N}^{(2)}$ is an $(N+1) \times(N+1)$ matrix carrying out the differentiation in $\tau$ composed by the Chebyshev differentiation matrices, $D_{N}^{(1)}$ where,

$$
\begin{gathered}
\left(D_{N}\right)_{00}=\frac{2 N^{2}+1}{6},\left(D_{N}\right)_{N N}=-\frac{2 N^{2}+1}{6}, \\
\left(D_{N}\right)_{j j}=\frac{-x_{j}}{2\left(1-x_{j}^{2}\right)}, j=1, \ldots, N-1, \\
\left(D_{N}\right)_{i j}=\frac{c_{i}}{c_{j}} \frac{(-1)^{i+j}}{\left(x_{i}-x_{j}\right)}, i \neq j, i, j=0, \ldots, N,
\end{gathered}
$$

and,

$$
c_{i}= \begin{cases}2 & \text { when } i=0 \text { or } N \\ 1 & \text { otherwise }\end{cases}
$$

and $D_{N}^{(2)}=D_{N}^{(1)} \circ D_{N}^{(1)}$. Finally, retaining a system of first-order ODEs in the $\rho$ co-ordinate we have the BVP formulation:

$$
\begin{aligned}
\left(u_{\rho}\right)_{i} & =w_{i}, \\
\left(w_{\rho}\right)_{i} & =-\frac{(d-1)}{\rho} w_{i}-\left(u_{\tau \tau}\right)_{i}+(1+\kappa) u_{i}-\left(u_{i} v_{i}\right), \\
\left(v_{\rho}\right)_{i} & =y_{i}, \\
\left(y_{\rho}\right)_{i} & =-\frac{(d-1)}{\rho} y_{i}-\delta\left(v_{\tau \tau}\right)_{i}+(\gamma+4 \kappa) v_{i}-\frac{1}{2}\left(u_{i}\right)^{2}, \\
\dot{\rho} & =1 .
\end{aligned}
$$

The numerical experiments in $[16,17,18,24]$ suggest that stable soliton solutions are even in both $\rho$ and $\tau$, respecting the reflectional symmetry of the equations $\rho \rightarrow-\rho$ and $\tau \rightarrow-\tau$. So we compute on the 1 st. quadrant and we re-write the differentiation matrix $A_{N}$ taking advantage of this symmetry.

We apply Neumann boundary conditions on $u$ and $v$ (i.e. $w_{i}=y_{i}=0$ ) at both ends of the domain since we wish to preserve symmetry at the origin and any linear boundary conditions at the far field end of the domain are known to exponentially converge to the solution if we take a large enough truncation of the domain, say $P$, such that $u$ and $v$ decay to zero numerically; see [10]. So for the BVP equations (3.5), we imposed the boundary conditions:

$$
\begin{aligned}
(w)_{i} & =0 \quad \text { and } \\
(y)_{i} & =0
\end{aligned}
$$

for $i=0, \ldots, N$ at $\rho=0$ and $\rho=P$. 
3.2. Initial Solution. The problem (3.5) and boundary conditions (3.6) is well set up for continuation of one-parameter families of solutions. First we need a starting solution at one particular parameter value.

To acquire good starting data we assume that $\delta=1, \kappa=0$ at which parameter values the equations (2.2) are radially symmetric. These symmetric solutions can be obtained from the reduced equations:

$$
\begin{aligned}
u_{r r}+\frac{d}{r} u_{r}-u+u v & =0, \\
v_{r r}+\frac{d}{r} v_{r}-\gamma v+\frac{1}{2} u^{2} & =0,
\end{aligned}
$$

where $r=\sqrt{\rho^{2}+\tau^{2}}$ is the radial co-ordinate. Taking $d=0$ and $\gamma=1$, equations (3.7) have an exact soliton solution [3], of the form:

$$
\begin{aligned}
& u(r)=\frac{3 \sqrt{2}}{2} \operatorname{sech}^{2}(r / 2), \\
& v(r)=\frac{3}{2} \operatorname{sech}^{2}(r / 2) .
\end{aligned}
$$

We can set up a simple continuation problem of solutions to (3.7), up to $d=1$; see Champneys et al. [6]. Once we have our new solution for $d=1$ we need to appropriately separate the data for the full continuation problem. Data for $u$ and $v$ is straight forward and the data for the first derivatives $w$ and $y$ are found by applying the chain rule to yield,

$$
\begin{aligned}
& w=u_{\rho}=\frac{\rho}{r} \frac{d u(r)}{d r}, \\
& y=v_{\rho}=\frac{\rho}{r} \frac{d v(r)}{d r},
\end{aligned}
$$

where $d u(r) / d r$ is the numerical continuation, computed derivative. Finally, the data needs to be interpolated to the Chebyshev grid in $\tau$.

3.3. Computation of Stability. Since we can now solve the BVP (3.5) to find the solitons, it would useful to know if the solitons found are stable or not. A standard approach to check the stability of stationary solitons is to use the Vakhitov-Kolokolov (VK) criterion; see Skryabin [21]. This VK criteria is a non-rigorous, necessary condition stating that solitons in Hamiltonian systems cannot be stable if $\partial_{\kappa} Q<0$, where $Q$ is the energy of the system and $\kappa$ is the effective propagation constant. The energy for this problem (2.2) being defined in (2+1)-dimensions as the integral $Q=\int_{-\infty}^{\infty} \int_{-\infty}^{\infty}\left(|u|^{2}+4|v|^{2}\right) d \tau d \rho$ and in (3+1)-dimensions, $Q=2 \pi \int_{-\infty}^{\infty} \int_{0}^{\infty}\left(|u|^{2}+4|v|^{2}\right) \rho d \tau d \rho$.

Integration with respect to $\tau$ is carried out by changing to Chebyshev coordinates to obtain the integral (in (2+1)-dimensions),

$$
Q=\int_{-\infty}^{\infty} \int_{-1}^{1}\left(|u(\rho, \tau)|^{2}+4|v(\rho, \tau)|^{2}\right)\left[\frac{\partial f^{-1}(\tau)}{\partial \tau}\right]^{-1} d x d \rho
$$

Noting that the internal integral is of the form $\int_{-1}^{1} p(x) d x$ and can be computed efficiently via ClenshawCurtis quadrature, see [25], we can append the BVP (3.5) and boundary conditions (3.6), with an integral condition:

$$
Q-q=0,
$$

where $Q$ is the energy of the system and $q$ is a 'free' continuation parameter. Using parameter continuation in both $\kappa$ and $q$, we can find where $\partial Q_{\kappa}=0$. This particular point presents a fold in the solution branch where we think of $q$ as the bifurcation parameter and $\kappa$ as part of the solution. Standard path-following techniques exist to follow fold points as another parameter, either $\gamma$ or $\delta$, is varied (see [20]) and hence trace out the VK stability criterion in two physical parameters. 
Since the VK criteria is found as the compatibility condition arising in the leading (zero) order asymptotic expansion of the linear eigenvalue problem governing the stability of the soliton (see [21]), the stability boundary only defines a necessary region for stability. Hence, we need to check its validity. In order to do this, we have implemented the above Chebyshev discretisation scheme in both the $\rho$ and $\tau$ co-ordinates, with a 4th order Runge-Kutta scheme (see $[25,2,5]$ for temporal discretisations) to simulate the full $\mathrm{z}$ dependent equations (2.1), as well as, calculating the linear spectrum directly. The data from the numerical continuation software is interpolated to a new mesh for use as an initial condition. For $\delta<0$ the end points of $u$ and $v$ in the $\tau$ direction are set to zero to stop Runge interpolation errors. All solutions shown have been interpolated onto a finer equi-spaced grid. It should be noted that the Runge-Kutta method is only suitable for solutions which do not have large changes in the z-derivatives since the PDEs are numerically stiff.

In order to calculate the linear spectrum of the PDEs (2.1) about the stationary soliton, we perturb the stationary soliton such that,

$$
\begin{aligned}
& u(\rho, \tau, z)=u_{s}(\rho, \tau, z)+e^{\lambda z}\left(\tilde{u}_{r}(\rho, \tau)+i \tilde{u}_{i}(\rho, \tau)\right) \quad \text { and } \\
& v(\rho, \tau, z)=v_{s}(\rho, \tau, z)+e^{\lambda z}\left(\tilde{v}_{r}(\rho, \tau)+i \tilde{v}_{i}(\rho, \tau)\right),
\end{aligned}
$$

where $u_{s}=u(\rho, \tau) e^{\kappa z i}, v_{s}=v(\rho, \tau) e^{2 \kappa z i}$ is the stationary soliton and $|\tilde{u}|,|\tilde{v}|<<1$ are small perturbations in the fundamental and second harmonic, respectively. Substituting the ansatz (3.11) in the PDEs (2.1) and retaining terms of first order in $\tilde{u}$ and $\tilde{v}$, we obtain the linear eigenvalue problem; see Buryak [3]. To carry out the 2-dimensional differentiation we have used Kronecker products with either Chebyshev or Fourier collocation matrices; see [25] for application of the Kronecker product to the 2D Laplace equation. Taking advantage of reflectional symmetry in the equations (2.1) we can reduce the size of the eigenvalue problem and hence make the problem tractable with out resorting to special matrix linear algebra techniques.

\section{Results.}

4.1. Implementation details. We have used the numerical continuation package AUTO [9] in our computations, see $[8,15,26]$ for other possible continuation software.

We have found that for a mesh of 11 points and $L=6$ in the $\tau$ co-ordinate and NTST $=10, N C O L=4$ in AUTO, in the $\rho$ co-ordinate, we have an error $\left(10^{-4}\right)$ in the maximum amplitude of $u$ and $v$ for $\delta=$ $1, \gamma=1, \kappa=0$ compared to AUTO computed ODEs (3.7) with NTST $=50$ and NCOL $=4$. For a mesh of 21 points $(L=6)$ the error decreases to $\left(10^{-6}\right)$. The stability boundaries computed on the two meshes differ quantitatively at the third decimal place, thought qualitatively they do not change; see Figures 4.2(a) and 4.2(c). Standard AUTO tolerances are used, see [9]. On a modern PC, computation of the stability boundaries, as in Figure 4.2(a), takes roughly 30 minutes. For numerical and interpolation reasons the infinite domain is truncated to $[-50,50]$.

Since we are using small grids in the order of $11 \times 11$ modes, we have implemented the Runge-Kutta scheme simulating the full z-dependent equations (2.1) and the linear stability analysis, in Matlab. We have found that using an $11 \times 11$ grid the computation of the eigenvalues takes less than a minute on a modern $\mathrm{PC}$ and going up to an $21 \times 21$ grid takes less than five minutes. Taking advantage of symmetry is important here to minimise the size of the linear eigenvalue problem in Matlab since the matrix grows like $4 N^{2}$. Typical mesh sizes are for the Fourier method $20 \times 20$ points over $[0,15]^{2}$ and the Chebyshev method $11 \times 11$ points with $L=6$. Again the AUTO computed solution is interpolated to the new mesh. Any other mesh sizes will be stated where used.

4.2. Continuation and Stability boundaries for $\delta \geq 0$ in $(2+1) D$. Embedding the above continuation scheme into AUTO we have explored the parameter region of the stationary solitons solutions to equations (2.1), as well as produced VK-stability boundaries for various mismatch parameters.

For $\delta \neq 1$ the solitons are known to be 'elliptic' in shape; see [17], where ellipticity is defined as the ratio of the soliton's temporal width to the transverse spatial width. In Figure 4.1, we see cross-sections of the computed light bullets for $\delta=0$ and $\delta=5$, along the spatial $(\rho)$ and temporal $(\tau)$ co-ordinate. The continuous lines show the cross-section of the soliton along the temporal co-ordinate with the dashed lines showing the cross-section along the spatial co-ordinate. From Figure 4.1, for the $\delta=5$, the dashed line occurs below the solid line, in both harmonics, showing expansion along the temporal co-ordinate relative 


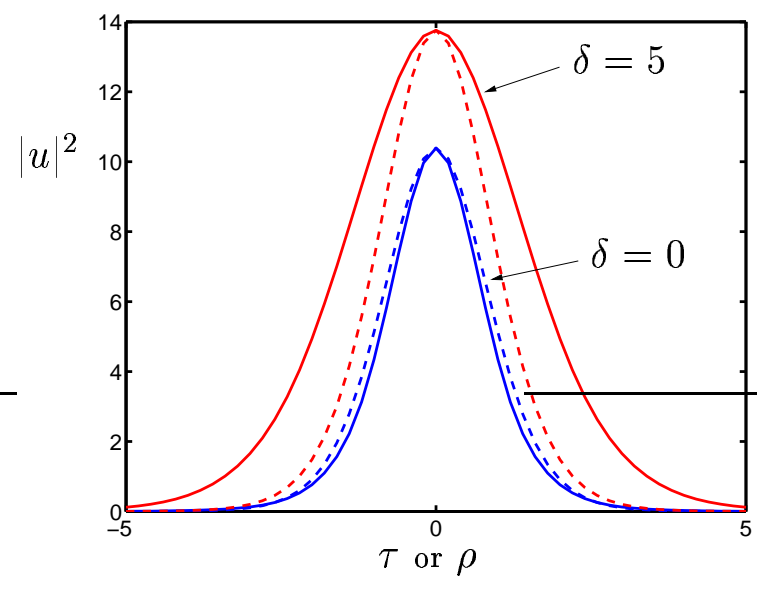

(a)

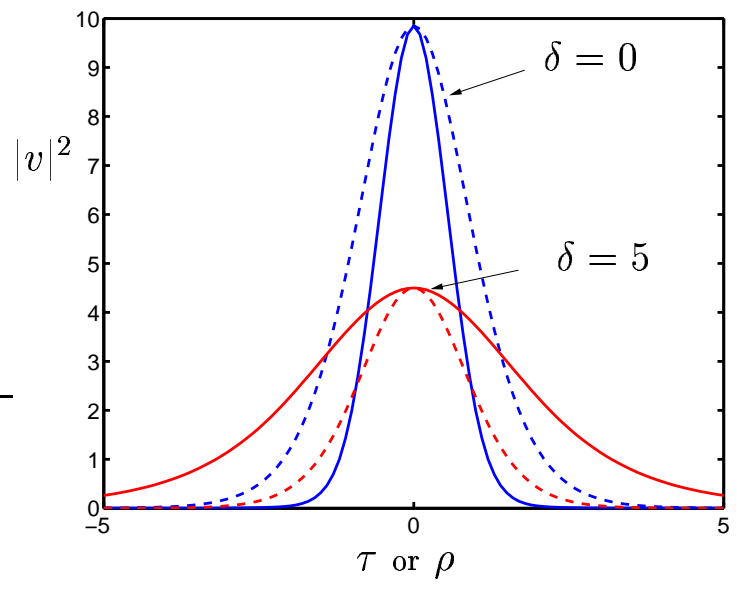

(b)

FIG. 4.1. Spatial and temporal sections of the light bullets along $\rho=0$ (solid line) and $\tau=0$ (dashed line), respectively, for $\gamma=1, \kappa=0$ in $(2+1) D$ and, $\delta=0$ and $\delta=5$. The fundamental harmonic is shown in (a) and the second harmonic is shown is (b).

to the transverse co-ordinate, whereas for $\delta=0$ we observe the opposite: the dashed line occurs above the solid line showing contraction along the temporal co-ordinate.

The VK-stability plots $\delta$ vs. $\kappa$ are shown in Figure 4.2. Consider first Figure 4.2(a). The region of stable solitons can be found in the top-right-hand corner of the Figure with the unstable region directly below. The stability boundary described by the VK criteria is shown as a line separating the stable and unstable regions. Beneath the unstable region we have drawn the line showing the existence boundary of the solitons governed by the bound $\kappa>\max \{-\gamma / 4,-1\}$ such that the tails in both harmonics decay exponentially. The region for the possible existence of 'quasi-solitons' is shown as $\delta<0$. A 'dip' in the stability boundary can be observed for mismatch parameters, $\gamma<4$, close to $\delta=0$ in $(2+1)$-dimensions. Qualitatively similar behaviour has been found by Malomed et al. [16] using a Gaussian approximation, albeit with gross quantitative differences. As $\gamma$ is increased towards 4, the stability boundary is found to uniformly approach the line $\kappa=-1(\delta>0)$, such that for $\gamma \geq 4$ all solitons are found to be stable; see Figure 4.2(e), numerically confirming and extending the result found by Pelinovsky et al. [19] and Steblina et al. [23]. For all $\gamma$ between 0 and 4 the stability boundary is found to have similar qualitative shape (results not depicted).

We can test the accuracy of the VK criteria by carrying out the linear stability analysis about the AUTO-computed solitons using our Chebyshev method. In Figure 4.2(a) we have drawn a dotted line taking a cross section through the VK-stability boundary at $\gamma=1$ and $\delta=1$. In Figure 4.3 we depict the maximum computed positive eigenvalue for $\gamma=1$ and $\delta=1 \mathrm{in}(2+1)$-dimensions for a range of $\kappa$ values. The VKstability boundary for these parameters occurs at $\kappa \approx-0.19$; shown in Figure 4.3 as a vertical dashed line. Using both the Chebyshev method and the Fourier method with a coarse mesh, described in $\S 3.3$, we see that the VK criteria gives an over-estimate for the stability region of the light bullets. Close to the stability boundary both the Chebyshev method and the Fourier method give similar results. Under higher resolution, the Chebyshev method shows the solitons losing stability closer to the VK-boundary. These results suggest (but do not prove) that in fact the VK criterion provides a sharp stability boundary.

Figure 4.4(a) shows an evolution plot of the intensity of the second harmonic of a stable stationary soliton along the line $\rho=0$. Just past the stability boundary at $\delta=1$ and $\kappa=-0.2$ we see from Figure 4.4(d) that the evolution of the stationary soliton, at this point, eventually becoming unstable after a long distance.

4.3. The region $\delta<0$ in $(\mathbf{2}+\mathbf{1}) \mathbf{D}$. For $\delta<0$, Towers et al. [24] showed that no true solitons exist in this parameter region. Instead one should be able to find solutions that do not decay at infinity but have oscillatory tails; known as quasi-solitons [14]. Using our numerical method we find (for small negative $\delta$ values), solitons with oscillating tails; see Figure 4.5 with the corresponding evolution plot shown in 
Figure 4.4(b) showing the quasi-soliton eventually becoming unstable. We have found, numerically, that one does not need go far into the quasi-soliton $(\delta<0)$ region to hit an existence boundary; e.g in $(2+1) \mathrm{D}$, $\kappa=-0.15$ and $\gamma=1$, the threshold for existence of such quasi-solitons appears to be $0>\delta \geq-0.042$. This may explain why Malomed et al. [16] found numerically stable quasi-solitons for small negative $\delta$, but no stable solitons far out into this region: they had stepped outside the existence boundary. As $\delta \rightarrow-0.042$ the fundamental harmonic tends to zero and the oscillations in the second harmonic grow.

If either $\gamma$ or $\kappa$ is increased the existence region appears to increase in size with some of these quasisolitons found to be numerically stable; see Figure 4.6. Computation of quasi-solitons using the Chebyshev basis on the infinite interval is known to poorly approximate oscillatory tails regardless of the number of modes used; see Boyd [1]. Several amendments to the Chebyshev method on the infinite domain have been suggested (see Boyd [1]) but the computation of quasi-solitons remains a challenging problem and has only been solved in (1+1)-dimensions; see Champneys et al. [7]. On should also stress that quasi-solitons have questionable physical validity since they require sources and sinks of energy at infinity. At best their existence represents parameter regimes where finite-time, almost localised structures may be expected to be observed in an experiment.

4.4. Stability boundaries in $(3+1) D$. If we assume radial symmetry in $(3+1)$-dimensions, we can reduce the equations to an effective $(2+1)$-dimension with the addition of a $(1 / \rho) d / d \rho$ term. This allows us to use our method and compute VK-stability boundaries in (3+1)-dimensions. (We shall not attempt to verify the VK-criteria in (3+1)D since results are already known, see Mihalache et al. [17].) The results here are to show the full 3-parameter shape of the stability boundary.

In (2+1)-dimensions it is known that solitons cannot be unstable for $\gamma \geq 4$, but in (3+1)-dimensions it is possible to find unstable regions for this parameter space; see Figure 4.2(f). This unstable region decreases in size until $\gamma=4$ where all solitons are found to be stable. As we pass through $\gamma=4$ we see the existence of a new unstable region. This region is always larger than the one found in $(2+1)$-dimensions for equivalent parameters; see Figures 4.2(a) and 4.2(b).

5. Conclusion. In this paper we have set out an efficient numerical method for finding stationary soliton solutions for the type-I second-harmonic-generation equations in (2+1)- and (3+1)-dimensions. The method described uses an adapted mesh applied to the localised soliton problem and Chebyshev differentiation matrices to carry out either the spatial or temporal differentiation. This adapted mesh method allows us to reduce the size of the discretised problem enabling the use of numerical continuation software. Using the Vakhitov-Kolokolov (VK) criterion we can trace out stability boundaries for various equation parameters in $(2+1)$ - and $(3+1)$-dimensions. We have also found, numerically, quasi-solitons when the dispersion at the second harmonic is normal. Known results for the ellipticity of the light bullets for various dispersion coefficients at the second harmonic and validity of the VK-criteria are confirmed, and extended to 3-parameter plots in $\delta, \kappa, \gamma$.

We have used our discretisation scheme to test the validity of the VK-stability criteria in $(2+1)$ dimensions via numerical simulation of the full z-dependent equations, as well as direct computation of the linear spectrum about the stationary soliton. Confirmation of the VK-stability criteria in (3+1)-dimensions can be found in [17].

Our results in (2+1)-dimensions confirm the findings of Mihalache et al. [17] and Skryabin et al. [22], yet in addition we have been able to provide a full two-parameter plot of the stability region and indeed how this region shrinks when we vary a third parameter.

Our numerical method can be extended for a wide range of soliton bearing equations including dissipative systems, though for dissipative systems the VK-criteria is no longer valid and so stability boundaries would have to be calculated another way. Future work will concentrate on the type-II interaction, second-harmonicgeneration equations (see [3]) where far less is known about the structure of the solutions in $(2+1)$ - and (3+1)-dimensions.

Acknowledgements. The authors would like to thank John P. Boyd, Boris A. Malomed and Dumitru Mihalache for their useful remarks. The authors would also like to thank Richard R. Kerswell for initially suggesting the numerical discretisation. 
[1] J. P. BoyD, Weakly Nonlocal Solitary Waves and Beyond-All-Orders Asymptotics: Generalised Solitons and Hyperasymptotic Perturbation Theory, Kluwer Academic Publishers, 1998.

[2] - Chebyshev and Fourier Spectral Methods, Dover Publishers, New York, 2nd. ed., 2001.

[3] A. V. BuRYAK, Solitons due to Quadratic Nonlinearities, PhD thesis, Australia National University, Canberra, December 181996.

[4] A. V. Buryak, P. D. Trapani, D. V. Skryabin, And S. Trillo, Optical solitons due to quadratic nonlinearities from basic ohysics to furturistic applications, Physics Reports, 370 (2002), pp. 63-235.

[5] C. Canuto, M. Hussaini, A. Quarteroni, and T. Zang, Spectral methods in Fluid Dynamics, Springer-Verlag, 1988.

[6] A. Champneys, Y. Kuznetsov, and B. Sandstede, A numerical toolbox for homoclinic bifurcation analysis, International Journal of Bifurcation and Chaos, 6 (1996), pp. 867-887.

[7] A. Champneys, J.-M. VAnden-Broek, And G. LORD, Do true elevation solitary waves exist? a numerical investigation., J. FLuid Mechanics, 454 (2002), pp. 403-417.

[8] A. Dhooge, W. Govaerts, And Y. Kuznetsov, Matcont: A matlab package for numerical bifurcation analysis of odes., ACM Trans. Math. Software, 29 (2003), pp. 141-164.

[9] E. Doedel, R. Paffenroth, A. Champneys, T. Fairgrieve, Y. Kuzetsov, B. Standstede, And X. Wang, Auto 2000: Continuation and bifurcation software for ordinary differential equations (with homcont), tech. report, Caltech, 2001.

[10] M. FRIEDMAN AND E. DOEDEL, Numerical computation and continuation of invariant manifolds connecting fixed points, SIAM Journal on Numerical Analysis, 28 (1991), pp. 789-808.

[11] H. He, M. WERnER, AND P. DRUMmOND, Simultaneous solitary-wave solutions in a nonlinear parametric waveguide, Physical Review E, 54 (1996), pp. 896-911.

[12] A. KanAShov AND A. RuBenchIK, On diffraction and dispersion effect on three wave interaction, Physica D, 4 (1981), pp. $122-134$.

[13] L. LiU, G. MoOre, AND R. D. Russell, Computation and continuation of homoclinic and heteroclinic orbits with arclength parameterization, SIAM Journal on Scientific Computing, 18 (1997), pp. 69-93.

[14] X. LIU, K. BECKWITT, AND F. WISE, Noncollinear generation of optical spatiotemporal solitons and application to ultrafast digital logic, Phys. Rev. E, 61 (2000), p. R4722.

[15] K. Lust, D. Roose, A. Spence, And A. Champneys., An adaptive newton-picard algorithm with subspace iteration for computing periodic solutions., SIAM Journal on Scientific Computing, 19 (1998), pp. 1188-1209.

[16] B. A. Malomed, P. Drummond, H. He, A. Bertson, D. Anderson, And M. Lisak, Spatiotemporal solitons in multidimensional optical media with a quadratic nonlinearity, Physical Review E, 56 (1997), pp. 4725-4735.

[17] D. Mihalache, D. Mazil, J. Dorring, And L. Torner, Elliptical light bullets, Optics Communications, 159 (1999), pp. $129-138$.

[18] D. Mihalache, D. Mazilu, L.-C. Crasovan, L. Torner, B. A. Malomed, And F. Lederer, Three-dimensional walking spatiotemporal solitons in quadratic media, Physical Review E, 62 (2000), pp. 7340-7347.

[19] D. Pelinovsky, A. Buryak, AND Y. Kivshar, Instability of solitons governed by quadratic nonlinearities, Physcial Review Letters, 75 (1995), pp. 591-595.

[20] R. SEYDEL, Nonlinear computation, International journal of bifurcation and chaos, 7 (1997), pp. $2105-2126$.

[21] D. V. SkRYABIN, Stability of multi-parameter solitons: asymptotic approach, Physica D, 139 (2000), pp. 186-193.

$[22]$ D. V. Skryabin AND W. J. FirTh, Generation and stability of optical bullets in quadratic nonlinear media, Optics Communications, 148 (1998), pp. 79-84.

[23] V. Steblina, Y. Kivshar, M. LiSAK, AND B. Malomed, Self-guided beams in a diffractive $\chi^{(2)}$ medium - variational approach, Optics Communications, 118 (1995), pp. 345-352.

[24] I. TOWERS, B. MALOMED, AND F. WISE, Light bullets in quadratic media with normal dispersion at the second harmonic, Physical Review Letters, 90 (2003), pp. 123902-1-123902-4.

[25] L. N. Trefethen, Spectral Methods in Matlab, SIAM, Philadelphia, 2000.

[26] F. UlRIKe AND J. WOLFGANG, Candys/qa - a software system for the qualitative analysis of nonlinear dynamical systems, International Journal for Bifurcation and Chaos, 2 (1992), pp. 773-794. 


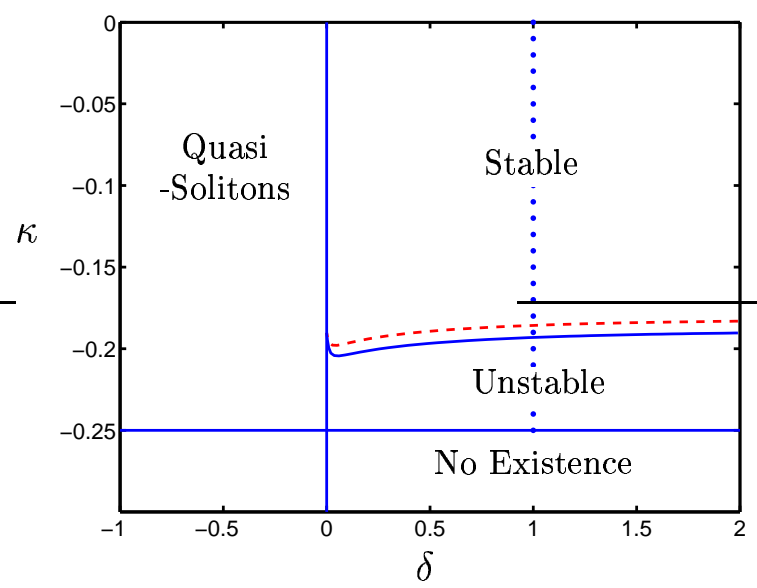

(a) $\gamma=1, D=2$

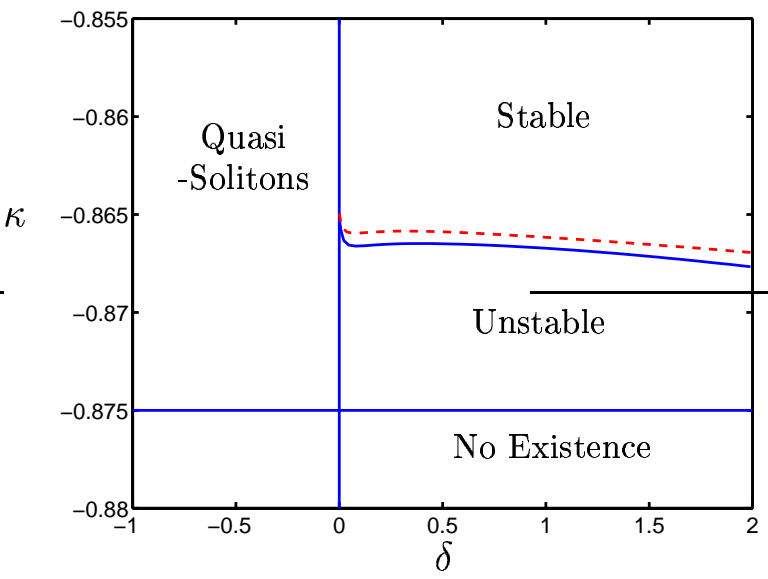

(c) $\gamma=3.5, D=2$

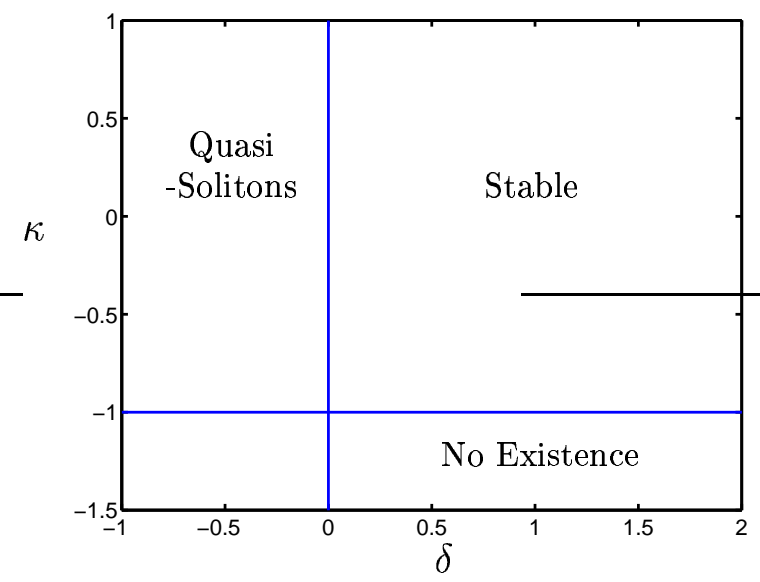

(e) $\gamma=5, D=2$

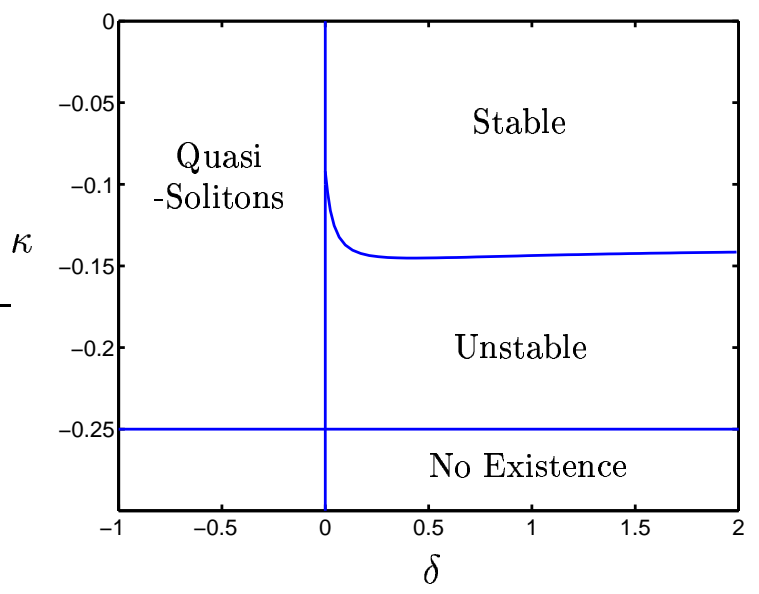

(b) $\gamma=1, D=3$

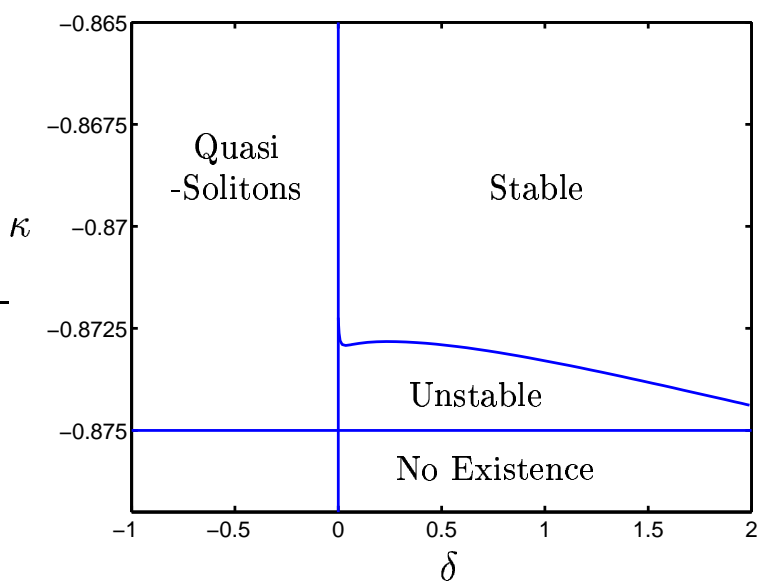

(d) $\gamma=3.5, D=3$

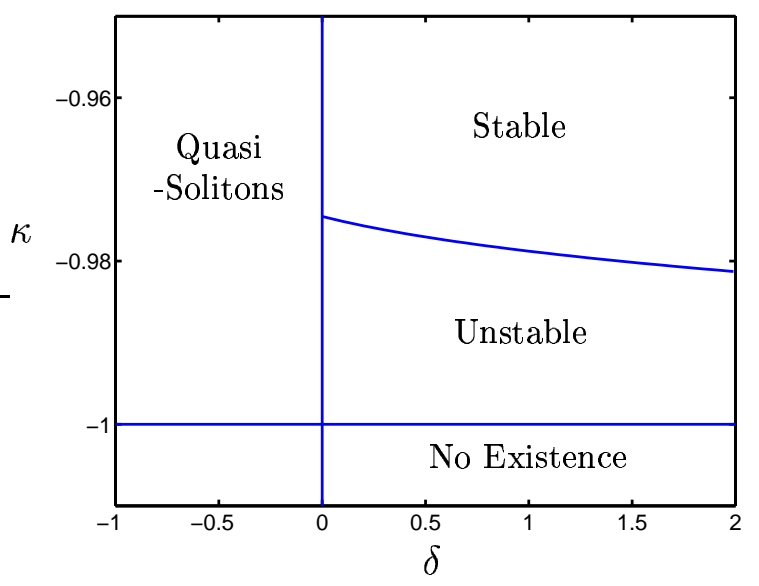

(f) $\gamma=5, D=3$

FIG. 4.2. Existence and stability boundaries for soliton solutions. The left-hand plots are in $(2+1) D(d=1)$ and righthand in $(3+1) D(d=2)$ The vertical dotted line in (a) represents the parameter path taken in Figure 4.3. In (a) and (c) the stability boundaries computed with 11 Chebyshev modes (solid line) and 21 Chebyshev modes (dashed line), $L=6$, are shown. 


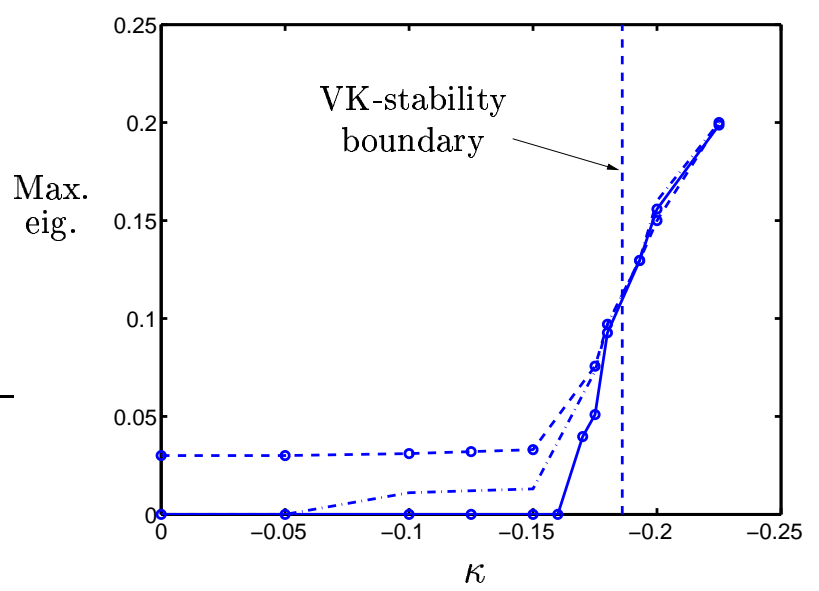

FIG. 4.3. Maximum eigenvalue computed from the linearisation about the stationary soliton for $\gamma=1$ and $\delta=1$. The $V K$-stability boundary is shown as a vertical dashed line at $\kappa=-0.186$ (calculated on a mesh of 21 points). The dashed line with circles corresponds to the linear spectrum computed with a Fourier method with an $20 \times 20$ grid over $[0,15]^{2}$ domain. The dashed-dot line corresponds to the linear spectrum being computed with the Chebyshev method with an $11 \times 11$ grid, $L=6$, and the solid line corresponds to a $21 \times 21$ grid with $L=13$. 


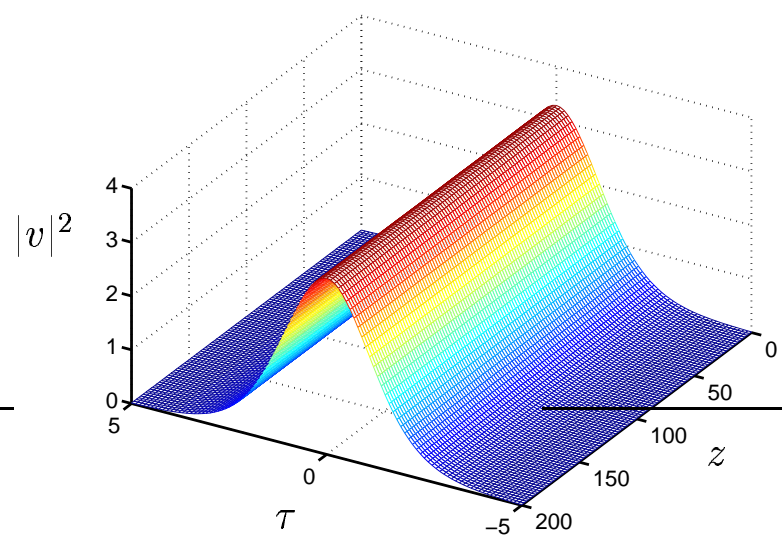

(a)



(c)

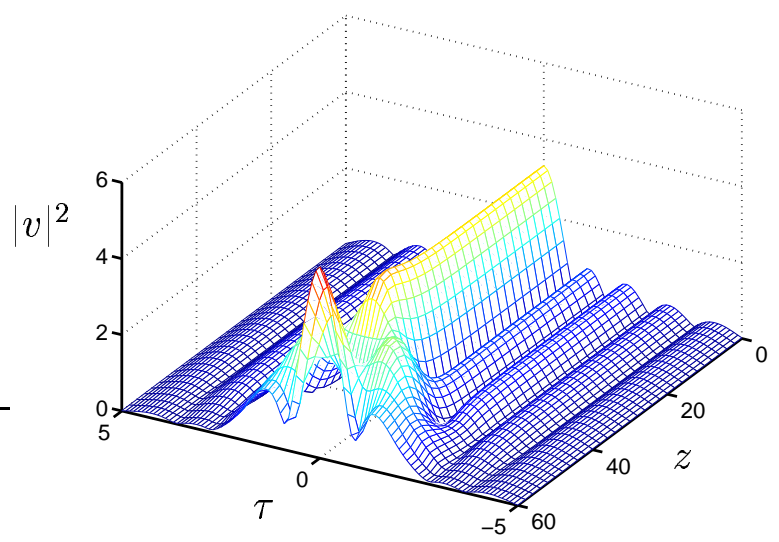

(b)

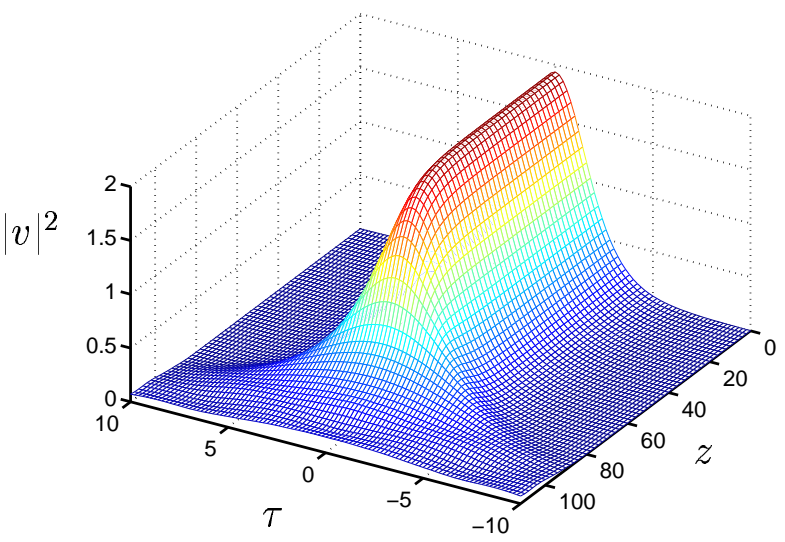

(d)

FIG. 4.4. Evolution plots of $|v(z, 0, \tau)|^{2}$ starting from initial data computed from numerical continuation for $\gamma=1$ and: (a) stable soliton $\delta=1$ and $\kappa=-0.15$, (b) unstable quasi-soliton $\delta=-0.04$ and $\kappa=-0.15$, (c) unstable soliton on stability boundary $\delta=1$ and $\kappa=-0.2$, (d) unstable soliton $\delta=1$ and $\kappa=-0.225$ 




(a)

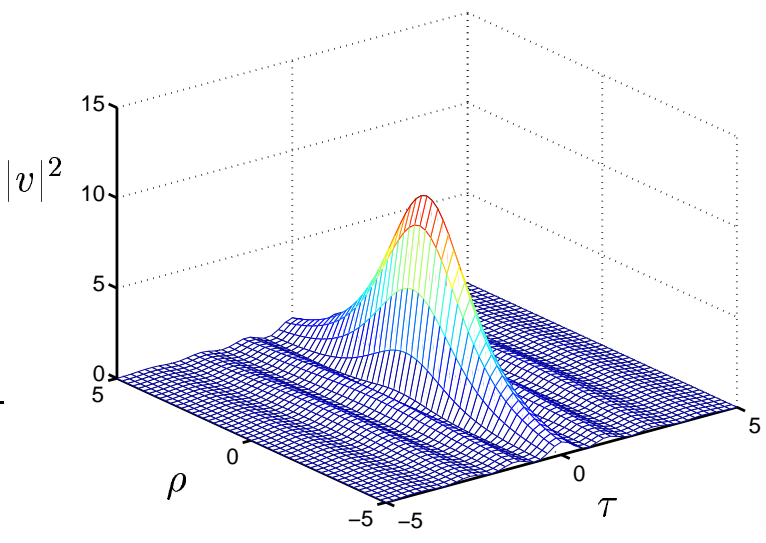

(b)

FIG. 4.5. Quasi-soliton for $\gamma=1, \kappa=-0.15$ and $\delta=-0.04$. Note the oscillations in the second harmonic $v$.

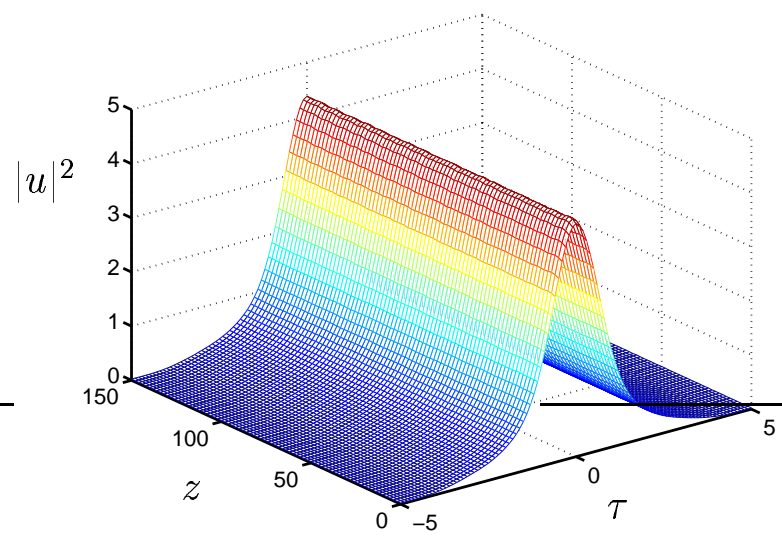

(a)

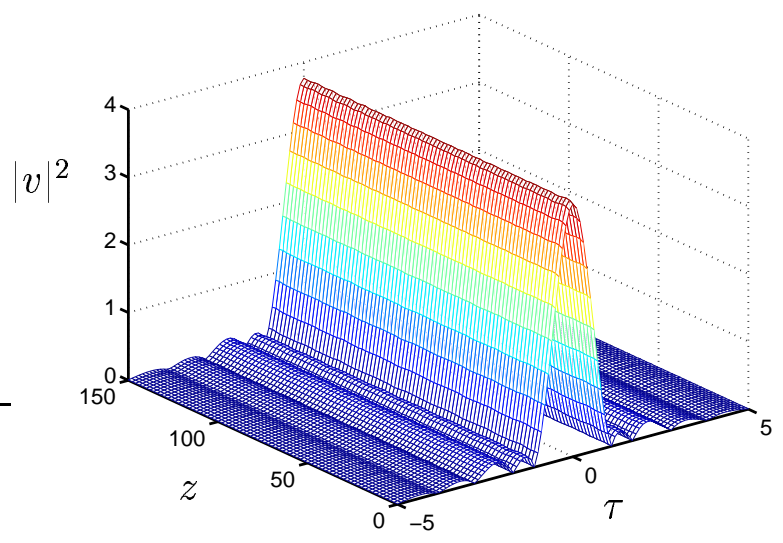

(b)

FIG. 4.6. Evolution of the (a) fundamental and (b) second harmonic for $\delta=-0.12, \gamma=2, \kappa=0$ in (2+1)D. This soliton is outside the stability boundary found by Towers et al. [24] 